\title{
REFLEXIVITY OF THE GROUP OF SURJECTIVE ISOMETRIES ON SOME BANACH SPACES
}

\author{
by LAJOS MOLNÁR* and BORUT ZALAR
}

(Received 8th June 1996)

To the memory of M.M.

\begin{abstract}
In this paper we study the problem of algebraic reflexivity of the isometry group of some important Banach spaces. Because of the previous work in similar topics, our main interest lies in the von Neumann - Schatten $p$-classes of compact operators. The ideas developed there can be used in $\ell_{p}$-spaces, Banach spaces of continuous functions and spin factors as well. Moreover, we attempt to attract the attention to this problem from general Banach spaces geometry view-point. This study, we believe, would provide nice geometrical results.
\end{abstract}

1991 Mathematics subject classification: 47B49, 47B04, 47B10.

\section{Introduction}

Let $X$ be a Banach space. Denote by $B(X)$ the algebra of all bounded linear operators acting on $X$. A subset $\mathcal{E} \subset B(X)$ is called algebraically [topologically] reflexive if the implication

$$
\begin{gathered}
T \in B(X), T x \in \mathcal{E} x(\forall x \in X) \Rightarrow T \in \mathcal{E} \\
{[T \in B(X), T x \in \overline{\mathcal{E} x}(\forall x \in X) \Rightarrow T \in \mathcal{E}]}
\end{gathered}
$$

holds true. This concept of reflexivity is very useful in the analysis of operator algebras (see [10] and the references therein). Most of the published work is about the reflexivity of derivation algebras and automorphism groups. For results on the algebraic reflexivity of derivation algebras see papers by Brešar, Kadison, Larson, Sourour and Šemrl $[3,4,8,11]$. A theorem, due to Shul'man, on the topological reflexivity of a derivation algebra acting on a $C^{*}$-algebra can be found in [17]. For results on algebraic reflexivity of the automorphism group see papers by Brešar and Šemrl [4] and [5]. In a recent article [13] of the first author it was proved that the automorphism group of

\footnotetext{
* The first author has been partially supported by the Hungarian National Foundation for Scientific Research (OTKA), Grant No. T-016846 F-019322 and MHB Bank, "A Magyar Tudományért" Foundation. The second author has been partially supported by the Slovene Ministry of Science.
} 
$B(H)$ is topologically reflexive. Moreover, it was also shown there that the automorphism group of $C(H)$ (the ideal of compact operators) is not topologically reflexive.

Another natural group, which makes sense not only for Banach algebras but for all Banach spaces as well, is the Banach-Lie group of all surjective (hence bijective) isometries. This group reflects the geometric properties of the underlying space. In [13] it was proved that the isometry group of $B(H)$ is algebraically and topologically reflexive. It was also shown that, similarly to the case of the automorphism group above, the isometry group of $C(H)$ is not topologically reflexive. The argument given there could actually be extended to all von Neumann-Schatten $p$-classes of compact operators so their isometry groups are topologically nonreflexive as well. In this paper we intend to investigate the reflexivity of the isometry group of some well-known, important Banach spaces.

Our paper is organized in three sections. The first section is the main one. Its purpose is to show that the isometry groups of von Neumann-Schatten $p$-classes are algebraically reflexive. As in many other results, the case $p=2$ is a trivial exception. Namely, it is an easy consequence of the definition that the isometry group of a Hilbert space $H$ is reflexive if and only if the dimension of $H$ is finite. The above mentioned result, together with the results in [13] establishes very natural examples of operator algebras for which the notions of algebraic and topological reflexivity differ. It also shows that for isometry groups of $C^{*}$-algebras one cannot hope to prove that they are topologically reflexive if and only if they are algebraically reflexive. This contrasts the notion of algebraic and topological irreducibility of $C^{*}$-algebras acting on Hilbert spaces where such theorem, due to Kadison's transitivity theorem, exists.

Since the problem of the algebraic reflexivity of isometry groups can be formulated for all Banach spaces, we devote the second section to study it in the context of some classical Banach spaces. However, we are able to give some positive results only for $\ell_{p}$-spaces and function spaces. This is because we can think of them as commutative analogues of von Neumann-Schatten classes so we are able to apply some ideas from the first section. This section, together with some open problems collected at the end of the paper, should be understood as our proposal for further investigations of the algebraic reflexivity problem of isometry groups from purely Banach space geometry view-point. We feel that the study in this generality could result in deep geometrical theorems.

Our work on operator algebras in the first section somehow naturally points to the directions of $J B^{*}$-triples. In $C^{*}$-theory there is only one family of type I von Neumann factors, namely $B(H)$ for different $H$. In $J B^{*}$-theory there are six families of such factors, $B(H)$ being only one of them. As mentioned before, the isometry group of $B(H)$ is algebraically reflexive. However, we are able, using the ideas of the first section, to find some type I von Neumann factors in the $J B^{*}$-category whose isometry groups are not algebraically reflexive. This shows that the algebraic reflexivity distinguishes type I von Neumann $J B^{*}$-factors, so our work could be of some interest in the $J B^{*}$-theory as well.

Let us fix the notation. In what follows, $H$ stands for a complex infinite dimensional separable Hilbert space. For any real number $1 \leq p<\infty$ we denote by $C_{p}(H)$ the von 
Neumann-Schatten $p$-class of compact operators acting on $H$. By definition $C_{\infty}(H)=C(H)$. Each of these spaces has a specific Banach algebra norm, called $p$-norm. When we are dealing with these spaces, we always consider $C_{p}(H)$ equipped with this $p$-norm. If $\Omega$ is a compact Hausdorff space, then $C(\Omega)$ denotes the Banach space of all continuous complex-valued functions on $\Omega$ with the usual sup-norm.

Finally, for the sake of a simplified writing, we use the following notion. If $X$ is a Banach space and $T \in B(X)$, then we say that $T$ is a locally surjective isometry if for every $x \in X$ there exists a surjective isometry $U_{x} \in B(X)$ such that $T x=U_{x} x$. With this notion the set of all surjective isometries of $X$ is algebraically reflexive if and only if every locally surjective isometry of $X$ is a surjective isometry. Note that in $[8,11,4,5]$ a similar terminology is used concerning the algebraic reflexivity of derivation algebras and isomorphism groups of operator algebras.

\section{Isometries of $C_{p}(H)$}

In this section we prove that the group of all surjective isometries of $C_{p}(H)$ is algebraically reflexive. This contrasts the previous result that the group of all surjective isometries on $C_{p}(H)$ is not topologically reflexive. During the proof of our result, the need for consideration of triple homomorphisms naturally arises. These mappings generalize the usual *-homomorphisms of operator algebras. They are well understood only in the case of triple isomorphisms. Possibly noninjective or nonsurjective triple homomorphisms could be important for the notion of direct limit in the category of $J B^{*}$-triples. In Theorem 1.5 we describe the general form of a triple homomorphism $\Phi: C_{p}(H) \rightarrow B(H)$. Using this result, in Theorem 1.6 we prove the algebraic reflexivity of the isometry group of $C_{p}(H)(p \neq 2)$. Recall that the Hilbert-Schmidt class $C_{2}(H)$ is a Hilbert space and hence every isometry on it is locally surjective.

It is a well-known result of Arazy [1] (for the simplest proof, see [7]) that for every surjective isometry $\Phi$ of $C_{p}(H)(p \neq 2)$, there exist unitaries $U, V$ and antiunitaries $U^{\prime}, V^{\prime}$ (conjugate-linear surjective isometries) on $H$ such that $\Phi$ is either of the form

$$
\Phi(A)=U A V \quad\left(A \in C_{p}(H)\right)
$$

or of the form

$$
\Phi(A)=U^{\prime} A^{*} V^{\prime} \quad\left(A \in C_{p}(H)\right)
$$

This result was extended by Sourour [18] to symmetric norm ideals which are not isomorphic to the Hilbert-Schmidt class $C_{2}$. A symmetric norm ideal is an ideal $\mathcal{J}$ of compact operators equipped with a Banach norm $v$ satisfying the following conditions

(i) $v(A)=\|A\|$ for all rank one operators $A$.

(ii) $v(U A V)=v(A)$ for all unitary $U, V$ and $A \in \mathcal{J}$.

(iii) Finite rank operators are dense in $\mathcal{J}$. 
In what follows, let $(\mathcal{J}, v)$ be a symmetric norm ideal, which is not isomorphic to $C_{2}$.

We begin with a few lemmas which are needed in the proofs of the main results of this section.

Lemma 1.1. Let $\Phi: \mathcal{J} \rightarrow \mathcal{J}$ be a locally surjective isometry. Then $\Phi$ is an isometry and it maps partial isometries into partial isometries.

Proof. From the form of surjective isometries of $\mathcal{J}$ it is easy to see that every such mapping preserves the set of partial isometries. Since the limit of a convergent sequence of partial isometries is also a partial isometry, we have the statement.

Lemma 1.2. If $\Phi: \mathcal{J} \rightarrow B(H)$ is a continuous linear mapping which preserves the set of all partial isometries in $\mathcal{J}$, then $\Phi$ is a triple homomorphism in the sense that

$$
\boldsymbol{\Phi}\left(A B^{*} A\right)=\boldsymbol{\Phi}(A) \boldsymbol{\Phi}(B)^{*} \Phi(A) \quad(A, B \in \mathcal{J}) .
$$

Proof. We shall say that the operators $A, B \in B(H)$ are orthogonal to each other if $A^{*} B=A B^{*}=0$ holds true. We first prove that $\Phi$ preserves the orthogonality between partial isometries. In fact, let $U, V$ be orthogonal partial isometries in $\mathcal{J}$. Let $\alpha \in \mathbb{C}$ be an arbitrary number of modulus 1 . It is easy to verify that $\alpha U+V$ is also a partial isometry. Let $P=\boldsymbol{\Phi}(U), Q=\boldsymbol{\Phi}(V)$. Since $\boldsymbol{\Phi}$ preserves the partial isometries, we obtain that

$$
\begin{gathered}
\alpha P+Q=(\alpha P+Q)(\alpha P+Q)^{*}(\alpha P+Q)= \\
\alpha P+Q+\left(P P^{*} Q+\alpha^{2} P Q^{*} P+Q P^{*} P\right)+\left(\alpha P Q^{*} Q+\bar{\alpha} Q P^{*} Q+\alpha Q Q^{*} P\right) .
\end{gathered}
$$

If we multiply the above equation by $\alpha$, it follows that

$$
\alpha^{3} P Q^{*} P+\alpha^{2}\left(P Q^{*} Q+Q Q^{*} P\right)+\alpha\left(P P^{*} Q+Q P^{*} P\right)+Q P^{*} Q=0
$$

holds for every $\alpha$ of modulus 1. Considering some particular values of $\alpha$, we obtain that

$$
P Q^{*} P=0, P Q^{*} Q+Q Q^{*} P=0, P P^{*} Q+Q P^{*} P=0, Q P^{*} Q=0 .
$$

Let us multiply the second equation by $P^{*}$ from the left. We obtain

$$
P^{*} P Q^{*} Q+\left(Q^{*} P\right)^{*}\left(Q^{*} P\right)=0
$$

Since $P, Q$ are partial isometries, it follows that the operators $P^{*} P$ and $Q^{*} Q$ are projections. From the equality above we infer that the product of these projections is selfadjoint and so they commute. But in this case their product is a positive operator. If we now take the equation $P^{*} P Q^{*} Q=-\left(Q^{*} P\right)^{*}\left(Q^{*} P\right) \leq 0$ into consideration, we get that $P^{*} P Q^{*} Q=0$. This implies that $Q^{*} P=0$. The relation $Q P^{*}=0$ can be obtained using a similar argument. 
Let $A \in \mathcal{J}$ be a finite rank operator. Then $A$ can be written as a finite linear combination of pairwise orthogonal partial isometries of finite rank. To see this, let $|A|=\sum_{i=1}^{n} \alpha_{i} P_{i}$ be the spectral resolution of the absolute value of $A$. Denote by $U$ the partial isometry corresponding to the polar decomposition $A=U|A|$. It is easy to verify that the operators $V_{i}=U P_{i}$ are pairwise orthogonal partial isometries and $A=\sum_{i=1}^{n} \alpha_{i} V_{i}$. Then we compute

$$
\begin{gathered}
\boldsymbol{\Phi}\left(A A^{*} A\right)=\Phi\left(\sum_{i=1}^{n} \alpha_{i}^{3} V_{i}\right)=\sum_{i=1}^{n} \alpha_{i}^{3} \Phi\left(V_{i}\right)= \\
\left(\sum_{i=1}^{n} \alpha_{i} \Phi\left(V_{i}\right)\right)\left(\sum_{i=1}^{n} \alpha_{i} \Phi\left(V_{i}\right)\right)^{*}\left(\sum_{i=1}^{n} \alpha_{i} \Phi\left(V_{i}\right)\right)=\boldsymbol{\Phi}(A) \boldsymbol{\Phi}(A)^{*} \boldsymbol{\Phi}(A) .
\end{gathered}
$$

Since $\Phi$ is continuous, we infer that

$$
\boldsymbol{\Phi}\left(A A^{*} A\right)=\boldsymbol{\Phi}(A) \boldsymbol{\Phi}(A)^{*} \Phi(A)
$$

holds for every $A \in \mathcal{J}$. Let us linearize this equation, i.e. replace $A$ by $A+B$. We have

$$
\begin{gathered}
\Phi\left(A A^{*} B+A B^{*} A+B A^{*} A+A B^{*} B+B A^{*} B+B B^{*} A\right)= \\
\boldsymbol{\Phi}(A) \boldsymbol{\Phi}(A)^{*} \boldsymbol{\Phi}(B)+\boldsymbol{\Phi}(A) \boldsymbol{\Phi}(B)^{*} \boldsymbol{\Phi}(A)+\boldsymbol{\Phi}(B) \boldsymbol{\Phi}(A)^{*} \boldsymbol{\Phi}(A)+ \\
\boldsymbol{\Phi}(A) \boldsymbol{\Phi}(B)^{*} \boldsymbol{\Phi}(B)+\boldsymbol{\Phi}(B) \boldsymbol{\Phi}(A)^{*} \boldsymbol{\Phi}(B)+\boldsymbol{\Phi}(B) \boldsymbol{\Phi}(B)^{*} \boldsymbol{\Phi}(A) .
\end{gathered}
$$

Let us replace $B$ by $\alpha B$ where $\alpha$ is an arbitrary real number. Considering some particular values of $\alpha$, it follows that

$$
\begin{gathered}
\boldsymbol{\Phi}\left(A A^{*} B+A B^{*} A+B A^{*} A\right)= \\
\boldsymbol{\Phi}(A) \boldsymbol{\Phi}(A)^{*} \boldsymbol{\Phi}(B)+\boldsymbol{\Phi}(A) \Phi(B)^{*} \boldsymbol{\Phi}(A)+\boldsymbol{\Phi}(B) \boldsymbol{\Phi}(A)^{*} \boldsymbol{\Phi}(A)
\end{gathered}
$$

If we replace $B$ by $i B$, respectively we multiply the above equation by $i$ and compare the obtained equations, we arrive at

$$
\boldsymbol{\Phi}\left(A B^{*} A\right)=\boldsymbol{\Phi}(A) \boldsymbol{\Phi}(B)^{*} \boldsymbol{\Phi}(A) \quad\left(A, B \in C_{p}(H)\right) .
$$

Remark. Triple homomorphisms represent an important class of morphisms on operator algebras. The reason is that every surjective isometry on a $C^{*}$-algebra (more generally, on a $J B^{*}$-triple) is a triple homomorphism $[9,6]$. Hence, one can deduce an algebraic property from a metrical one. This is a very important step, since the -algebraic properties are usually much easier to handle than the metrical ones.

Lemma 1.3. If $\left(U_{n}\right)$ is an arbitrary family of pairwise orthogonal partial isometries, then the series $\sum_{n} U_{n}$ converges strongly to an operator $T \in B(H)$. Moreover, $T$ is also a partial isometry. 
Proof. Let $x \in H$. We have

$$
\left\langle U_{n} x, U_{m} x\right\rangle=0 \quad(n \neq m)
$$

Since $U_{n}^{*} U_{n}$ are pairwise orthogonal projections, we infer that

$$
\left\|U_{n} x\right\|^{2}=\left\langle U_{n}^{*} U_{n} x, x\right\rangle=\left\|U_{n}^{*} U_{n} x\right\|^{2}
$$

and so $\sum_{n}\left\|U_{n} x\right\|^{2}<\infty$. This gives the convergence of the series $\sum_{n} U_{n} x(x \in H)$. Moreover, since every finite partial sum of $\sum_{n} U_{n}$ is a partial isometry we obtain that the family of these partial sums is uniformly bounded. The first assertion now follows. It remains to prove that $T$ is a partial isometry. Using the strong convergence of $\sum_{n} U_{n}$, it is easy to see that $\sum_{n} U_{n}^{*} U_{n}$ converges weakly to $T^{*} T$. But the terms in $\sum_{n} U_{n}^{*} U_{n}$ are pairwise orthogonal projections. Therefore, this series converges strongly to $T^{*} T$. Since the multiplication is continuous on every bounded subset of $B(H)$ with respect to the strong operator topology, hence we obtain that $\sum_{n} U_{n} U_{n}^{*} U_{n}=T T^{*} T$ in the strong operator topology. But $U_{n} U_{n}^{*} U_{n}=U_{n}$ holds for every $n$ and thus we have $T T^{*} T=T$, i.e. $T$ is a partial isometry.

Lemma 1.4. Let $\Phi: \mathcal{J} \rightarrow B(H)$ be a continuous triple homomorphism. Let $\left(P_{n}\right)$ be a maximal family of pairwise orthogonal rank-one projections in $B(H)$ and denote $T=\sum_{n} \boldsymbol{\Phi}\left(P_{n}\right)$. Then the mapping $\Psi: \mathcal{J} \rightarrow B(H)$ defined by

$$
\Psi(A)=T^{*} \Phi(A) \quad(A \in \mathcal{J})
$$

is a continuous Jordan *-homomorphism on J. Moreover, we have

$$
\Phi(A)=T \Psi(A) \quad(A \in \mathcal{J})
$$

Proof. Obviously, $\Phi$ preserves the partial isometries and, by the proof of Lemma 1.2, their orthogonality as well. Consequently, by Lemma 1.3, $T$ is a well-defined partial isometry. Let $Q=Q_{n}$ be the $n$th partial sum of $\sum_{k} \Phi\left(P_{k}\right)$, i.e. let $Q=Q_{n}=\sum_{k=1}^{n} \Phi\left(P_{k}\right)$. We define $\mathcal{A}_{Q}=\left\{A \in C_{p}(H): Q A Q=A\right\}$. Let $A \in \mathcal{A}_{Q}$. If $m>n$, then

$$
\Phi\left(P_{m}\right)^{*} \Phi(A)=\Phi\left(P_{m}\right)^{*} \Phi(Q) \Phi\left(A^{*}\right)^{*} \Phi(Q)=0
$$

Therefore, we have

$$
\Psi(A)=T^{*} \Phi(A)=\Phi(Q)^{*} \Phi(A)
$$

If we linearize the equation characterizing triple homomorphisms in $A$, we obtain that

$$
\Phi\left(A B^{*} C+C B^{*} A\right)=\boldsymbol{\Phi}(A) \Phi(B)^{*} \Phi(C)+\Phi(C) \Phi(B)^{*} \Phi(A)
$$


From (1) we have

$$
2 \Phi(A)=\Phi(A) \Phi(Q)^{*} \Phi(Q)+\Phi(Q) \Phi(Q)^{*} \Phi(A) .
$$

Using the fact that $\Phi(Q)^{*} \Phi(Q)$ and $\Phi(Q) \Phi(Q)^{*}$ are projections, it is an easy algebraic manipulation to prove that the above equation implies

$$
\boldsymbol{\Phi}(A) \boldsymbol{\Phi}(Q)^{*} \boldsymbol{\Phi}(Q)=\boldsymbol{\Phi}(Q) \boldsymbol{\Phi}(Q)^{*} \boldsymbol{\Phi}(A)=\boldsymbol{\Phi}(A) .
$$

For an arbitrary $A \in \mathcal{A}_{Q}$ we now compute

$$
\begin{gathered}
\boldsymbol{\Psi}\left(A^{2}\right)=\boldsymbol{\Phi}(Q)^{*} \boldsymbol{\Phi}\left(A^{2}\right)=\boldsymbol{\Phi}(Q)^{*} \boldsymbol{\Phi}\left(A Q^{*} A\right)= \\
\boldsymbol{\Phi}(Q)^{*} \boldsymbol{\Phi}(A) \boldsymbol{\Phi}(Q)^{*} \boldsymbol{\Phi}(A)=\left(\boldsymbol{\Phi}(Q)^{*} \boldsymbol{\Phi}(A)\right)^{2}=\boldsymbol{\Psi}(A)^{2}
\end{gathered}
$$

and using (2) we obtain

$$
\begin{gathered}
\boldsymbol{\Psi}\left(A^{*}\right)=\boldsymbol{\Phi}(Q)^{*} \boldsymbol{\Phi}\left(A^{*}\right)=\boldsymbol{\Phi}(Q)^{*} \boldsymbol{\Phi}\left(Q A^{*} Q\right)= \\
\boldsymbol{\Phi}(Q)^{*} \boldsymbol{\Phi}(Q) \boldsymbol{\Phi}(A)^{*} \boldsymbol{\Phi}(Q)=\boldsymbol{\Phi}(A)^{*} \boldsymbol{\Phi}(Q)=\boldsymbol{\Psi}(A)^{*}
\end{gathered}
$$

The equations (3) and (4) give us that $\Psi$ is a Jordan "-homomorphism on $\mathcal{A}_{Q}$. Now, the first statement follows from the continuity of $\Psi$ together with the fact that for every $A \in \mathcal{J}$ the sequence $\left(Q_{n} A Q_{n}\right)$ converges to $A$ in $\mathcal{J}$.

Let us consider the last assertion. From (4) we know that

$$
\boldsymbol{\Phi}(Q) \Phi(Q)^{*} \Phi(A)=\boldsymbol{\Phi}(A) \quad\left(A \in \mathcal{A}_{Q}\right)
$$

This gives us first that

$$
T \boldsymbol{\Phi}(Q)^{*} \Phi(A)=\boldsymbol{\Phi}(A) \quad\left(A \in \mathcal{A}_{Q}\right)
$$

and then that

$$
T T^{*} \Phi(A)=\Phi(A) \quad(A \in \mathcal{J})
$$

Since $T^{*} \Phi(A)=\Psi(A)$, the proof is complete.

Theorem 1.5. Let $\Phi: \mathcal{J} \rightarrow B(H)$ be a continuous triple homomorphism. Then $\Phi$ is of the form

$$
\Phi(A)=\sum_{\alpha} U_{\alpha}^{\prime} A U_{\alpha}^{*}+\sum_{\beta} V_{\beta}^{\prime} A^{*} V_{\beta}^{*} \quad\left(A \in C_{p}(H)\right)
$$

where $U_{\alpha}, U_{\alpha}^{\prime}$ are isometries, $V_{\beta}, V_{\beta}^{\prime}$ are antiisometries such that the ranges of the operators $U_{\alpha}, V_{\beta}$ form a pairwise orthogonal family and the same holds true for the ranges of the operators $U_{\alpha}^{\prime}, V_{\beta}^{\prime}$. 
Proof. We use the notation of Lemma 1.4. So, we have a continuous Jordan "homomorphism $\boldsymbol{\Psi}: \mathcal{J} \rightarrow B(H)$. Observe that $\boldsymbol{\Psi}$ is continuous on $F(H)$ with respect to the operator norm topology. Indeed, just as in the proof of the previous lemma one can verify that $\Psi_{I F(H)}$ preserves the positive operators and hence it preserves the order. Let $A \in F(H)$ be an arbitrary positive operator. Then we have $A^{2} \leq\|A\| A$. Consequently, we obtain

$$
\Psi(A)^{2} \leq\|A\| \Psi(A)
$$

Since $\Psi(A) \geq 0$, we infer that $\|\Psi(A)\|^{2} \leq\|A\|\|\Psi(A)\|$. Therefore, $\|\Psi(A)\| \leq\|A\|$ holds true for every positive operator $A \in F(H)$. If $A \in F(H)$ is self-adjoint, then we compute

$$
\|\Psi(A)\|^{2}=\left\|\Psi(A)^{2}\right\|=\left\|\Psi\left(A^{2}\right)\right\| \leq\left\|A^{2}\right\|=\|A\|^{2}
$$

Since the operations of taking real as well as imaginary parts of operators are continuous in the operator norm topology, we have the continuity of $\Psi_{\mid F(H)}$. This implies that there is a unique continuous Jordan *-homomorphism $J$ on $C(H)$ that extends $\Psi_{\mid F(H)}$. Since the norm $v$ majorizes the operator norm, it is clear that $J_{\mid \mathcal{J}}=\Psi$. $\left[19\right.$, Lemma 3.1] states that every Jordan *-homomorphism of a $C^{*}$-algebra into a von Neumann algebra has an ultra-weakly continuous extension onto the second dual which is also a Jordan "-homomorphism. The second dual of $C(H)$ is $B(H)$. Let $\tilde{J}: B(H) \rightarrow B(H)$ denote the corresponding extension of $J$.

$[19$, Lemma 3.2] states that if $J$ is a Jordan *-homomorphism of a von Neumann algebra into the algebra of all bounded operators acting on a Hilbert space, then there exist central projections $E, F$ in the $C^{*}$-algebra generated by the range of $J$ such that $E+F=I$ and the mappings

$$
A \mapsto J(A) E, \quad A \mapsto J(A) F
$$

are a "-homomorphism and a "-antihomomorphism, respectively. It is a well-known result of Arveson [2, Proposition 2.1] that on $B(H)$ every normal *-endomorphism is of the form

$$
A \mapsto \sum_{\alpha} U_{\alpha} A U_{\alpha}^{*}
$$

where the operators $U_{\alpha}$ are isometries with pairwise orthogonal ranges. Similarly, every normal *-antiendomorphism of $B(H)$ is of the form

$$
A \mapsto \sum_{\beta} V_{\beta} A^{*} V_{\beta}^{*},
$$

where the operators $V_{\beta}$ are antiisometries with pairwise orthogonal ranges. 
Let us now consider our Jordan "endomorphism $\bar{J}$ on $B(H)$. Using the results mentioned above, it follows that $\tilde{J}$ can be written in the form

$$
A \mapsto \sum_{z} U_{z} A U_{\alpha}^{*}+\sum_{\beta} V_{\beta} A^{*} V_{\beta}^{*}
$$

where $\left\{U_{\alpha}\right\}$ is a family of isometries and the elements of $\left\{V_{\beta}\right\}$ are antiisometries such that the whole family $\left\{U_{\alpha}, V_{\beta}\right\}$ consists of operators having pairwise orthogonal ranges.

Obviously, we have

$$
\Psi(A)=\sum_{\alpha} U_{\alpha} A U_{\alpha}^{*}+\sum_{\beta} V_{\beta} A^{*} V_{\beta}^{*} \quad(A \in \mathcal{J})
$$

From Lemma 1.4 we obtain that

$$
\Phi(A)=\sum_{\alpha} T U_{\alpha} A U_{\alpha}^{*}+\sum_{\beta} T V_{\beta} A^{*} V_{\beta}^{*} \quad(A \in \mathcal{J})
$$

It remains to prove that $T U_{\alpha}$ are isometries, $T V_{\beta}$ are antiisometries and the family $\left\{U_{\alpha}, V_{\beta}\right\}$ consists of operators having pairwise orthogonal ranges. It is obvious that

$$
\left(T U_{x}\right)^{*}\left(T U_{x}\right)=U_{\alpha}^{*} T^{*} T U_{x}
$$

We have obtained in the proof of Lemma 1.3 that $T^{*} T=\sum_{n} \boldsymbol{\Phi}\left(P_{n}\right)^{*} \boldsymbol{\Phi}\left(P_{n}\right)$, where this series converges strongly. Moreover,

$$
\Phi\left(P_{n}\right)^{*} \Phi\left(P_{n}\right)=T^{*} \Phi\left(P_{n}\right)=\Psi\left(P_{n}\right)=\sum_{\alpha} U_{\alpha} P_{n} U_{\alpha}^{*}+\sum V_{\beta} P_{n} V_{\beta}^{*}
$$

Using the pairwise orthogonality of the ranges of $U_{\alpha}, V_{\beta}$, we infer from (6) that

$$
\left(T U_{\alpha}\right)^{*}\left(T U_{\alpha}\right)=\sum_{n} U_{x}^{*} U_{a} P_{n} U_{\alpha}^{*} U_{x}=\sum_{n} P_{n}=I
$$

The relation $\left(T V_{\beta}\right)^{*}\left(T V_{\beta}\right)=I$ can be proved in a similar way. Therefore, the operators $T U_{\alpha}, T V_{\beta}$ are isometries and antiisometries, respectively. Moreover, the same argument can be applied to verify that, for example,

$$
\left(T U_{\alpha}\right)^{*}\left(T V_{\beta}\right)=U_{\alpha}^{*} T^{*} T V_{\beta}=0
$$

holds true. The pairwise orthogonality of the ranges of operators which belong to the family $\left\{T U_{2}, T V_{\beta}\right\}$ is now easy to see.

Remark. The converse of the statement of our previous theorem is also true. We 
mean that every mapping of the form (5) is a continuous triple homomorphism. This can be verified by an elementary computation.

Proposition 1.6. Let $(\mathcal{J}, v)$ be a symmetric norm ideal. Then the isometry group of $\mathcal{J}$ is algebraically reflexive unless $\mathcal{J}$ is isomorphic to the Hilbert-Schmidt class.

Proof. Let $\Phi: \mathcal{J} \rightarrow \mathcal{J}$ be a linear mapping which is a locally surjective isometry. Since the norm $v$ dominates the operator norm, this mapping is continuous when viewed as a mapping $\Phi: \mathcal{J} \rightarrow B(H)$. It follows from what we have proved above that $\Phi$ is of the form

$$
\Phi(A)=\sum_{\alpha} U_{\alpha}^{\prime} A U_{\alpha}^{*}+\sum_{\beta} V_{\beta}^{\prime} A^{*} V_{\beta}^{*} \quad(A \in \mathcal{J})
$$

where $U_{\alpha}, U_{\alpha}^{\prime}$ are isometries, $V_{\beta}, V_{\beta}^{\prime}$ are antiisometries such that the ranges of the operators $U_{\alpha}, V_{\beta}$ forms a pairwise orthogonal family and the same holds true for the ranges of the operators $U_{x}^{\prime}, V_{\beta}^{\prime}$. But since $\Phi$ is locally surjective, it follows from the form of the surjective isometries of $\mathcal{J}$ that $\Phi$ maps rank-one operators into rank-one operators. It implies that the right side of (7) reduces to only one term. So, let us suppose that, for example, $\Phi$ is of the form

$$
A \mapsto U^{\prime} A U^{*}
$$

where $U^{\prime}, U$ are isometries. Since $\Phi$ is a locally surjective isometry, we easily have that $\Phi(A)$ has dense range whenever $A$ has. Therefore $U^{\prime}$ is surjective. Similarly, if $A$ is injective, then $\Phi(A)$ is also injective which implies that $U^{*}$ is injective. It follows that $U, U^{\prime}$ are unitaries and, consequently, $\Phi$ is surjective. This completes the proof.

Theorem 1.7. If $p \neq 2$, then the group of all surjective isometries of $C_{p}(H)$ is algebraically reflexive.

\section{Isometries of $\ell_{p}$-spaces and function spaces}

In some sense, the commutative counterparts of the $C_{p}(H)$ spaces are the classical $\ell_{p}$-spaces $(1 \leq p<\infty), c_{0}$ corresponds to $C(H)$ and $\ell_{\infty}$ is the commutative analogue of $B(H)$. In what follows we consider our problem for these spaces and we prove that, with the trivial exception $p=2$, the groups of their surjective isometries are also algebraically reflexive.

Let us first describe the surjective isometries of these Banach spaces. We begin with $\ell_{p}(1 \leq p<\infty, p \in 2)$. Following the same argument that was carried out to determine the surjective isometries of $L_{p}[0,1]$ in $[15,15$. Theorem (Lamperti), p. 275], one can see that every surjective isometry on each of the spaces $\ell_{p}(1 \leq p<\infty, p \in 2)$ is of the form 


$$
f \mapsto \tau \cdot f \circ \varphi
$$

where $\tau$ is a sequence with terms of modulus 1 and $\varphi: \mathbb{N} \rightarrow \mathbb{N}$ is a bijection.

Since $c_{0}$ can be considered as the algebra of all continuous complex-valued functions defined on a locally compact Hausdorff space and vanishing at infinity, by the wellknown Banach-Stone theorem it follows that the surjective isometries of $c_{0}$ are also of the form (9).

To determine the surjective isometries of the remaining space $\ell_{\infty}$, let $\beta \mathbb{N}$ stand for the Stone- $\breve{C}$ ech compactification of $\mathbb{N}$. Every function $f \in \ell_{\infty}$ has a unique extension $\tilde{f} \in C(\beta \mathbb{N})$ having the same norm as $f \in \ell_{\infty}$ has. The mapping

$$
f \mapsto \tilde{f}
$$

is an isometric isomorphism between $\ell_{\infty}$ and $C(\beta \mathbb{N})$. Let $\Phi: \ell_{\infty} \rightarrow \ell_{\infty}$ be a surjective isometry. Using the Banach-Stone theorem again, we infer that

$$
\Phi(f)(n)=\tilde{\tau}(n) \cdot \tilde{f}(\tilde{\varphi}(n)) \quad(n \in \mathbb{N})
$$

where $\bar{\tau}: \beta \mathbb{N} \rightarrow \mathbb{C}$ is a continuous function with values of modulus 1 and $\bar{\varphi}: \beta \mathbb{N} \rightarrow \beta \mathbb{N}$ is a homeomorphism. Since the element of $\mathbb{N}$ are isolated in $\beta \mathbb{N}$ and the elements of $\beta \mathbb{N} \backslash \mathbb{N}$ are not, we get that the restriction $\varphi$ of $\tilde{\varphi}$ onto $\mathbb{N}$ is a bijection of $\mathbb{N}$. Consequently, we arrive at

$$
\Phi(f)(n)=\tau(n) \cdot f(\varphi(n)) \quad(n \in \mathbb{N})
$$

where $\tau=\tilde{\eta}_{\ell_{\infty}}$. This means that the surjective isometries of $\ell_{\infty}$ are also of the form (9).

Theorem 2.1. The groups of all surjective isometries of the spaces $\ell_{\infty}(1 \leq p \leq \infty, p \neq 2)$ and $c_{0}$ are algebraically reflexive.

Proof. Let $\mathcal{A}$ be any of the spaces $\ell_{p}(1 \leq p<\infty, p \neq 2), c_{0}$. Consider a locally surjective isometry $\Phi: \mathcal{A} \rightarrow \mathcal{A}$. Let $\left(e_{n}\right)$ denote the standard base in $\mathcal{A}$ which means that $c_{n}$ is the sequence whose terms are all 0 with the exception of the $n$th one which is 1. Since $\Phi$ is a locally surjective isometry, by (9) we obtain that for every $n \in \mathbb{N}$ there exist a $k(n) \in \mathbb{N}$ and a complex number $\lambda_{k(n)}$ with modulus 1 such that $\Phi\left(e_{n}\right)=\lambda_{k(n)} e_{k(n)}$. Let $n \neq m$. From $\Phi\left(e_{n}+e_{m}\right)=\Phi\left(e_{n}\right)+\Phi\left(e_{m}\right)$ and (9) we infer that $k(n) \neq k(m)$. We next assert that $k: \mathbb{N} \rightarrow \mathbb{N}$ is a surjective function. To this end, let us consider a sequence $\left(\alpha_{n}\right) \in \mathcal{A}$ with nonzero terms. Using the equality

$$
\Phi\left(\sum_{n} \alpha_{n} e_{n}\right)=\sum_{n} \alpha_{n} \Phi\left(e_{n}\right)
$$

and the form (9) of surjective isometries, it follows that the sequence on the left side 
of (10) has only nonzero terms. This gives the surjectivity of $k$. We then easily obtain that the range of $\Phi$ contains every cofinite sequence. Since $\Phi$ is an isometry, we have the surjectivity of $\Phi$.

We now consider the case of $\ell_{\infty}$. The same argument that was followed above (the only exception is that we choose $\left.\left(\alpha_{n}\right) \in c_{0}\right)$ yields that the range of $\Phi$ contains every cofinite sequence. Plainly, $\ell_{\infty}$ is a unital $C^{*}$-algebra and, by (9), $\Phi$ maps the unitary elements of $\ell_{\infty}$ into unitary elements. A well-known theorem of Russo and Dye [16, Corollary 2] asserts that every unitary preserving mapping on a unital $C^{*}$-algebra is a Jordan *-homomorphism multiplied by a fixed unitary element. This gives us that there is a fixed sequence $\tau \in \ell_{\infty}$ with terms of modulus 1 and a Jordan *-homomorphism $J: \ell_{\infty} \rightarrow \ell_{\infty}$ such that $\Phi(f)=\tau \cdot J(f)\left(f \in \ell_{\infty}\right)$. We obtain that the range of $J$ contains every cofinite sequence. But it was proved for an arbitrary ${ }^{*}$-homomorphism of $\ell_{\infty}$ that if its range contains the cofinite sequences, then it is automatically surjective [14, Theorem 3]. This completes the proof of the theorem.

In the following theorem we solve our reflexivity problem for $C(\Omega)$ under a not too restrictive condition on $\Omega$. We recall that a topological space is called first countable if each of its points has countable neighbourhood base.

Theorem 2.2. Let $\Omega$ be a first countable compact Hausdorff space. Then the group of all surjective isometries of $C(\Omega)$ is algebraically reflexive.

Proof. Let $\Phi: C(\Omega) \rightarrow C(\Omega)$ be a locally surjective isometry. Similarly to the proof of Theorem 2.1, by the Banach-Stone theorem it is easy to see that $\Phi$ maps continuous functions with modulus 1 into functions with modulus 1 . Applying the theorem of Russo and Dye [16, Corollary 2] again, we obtain $\Phi$ is a ${ }^{*}$-homomorphism multiplied by a fixed continuous function with modulus 1 . Without any loss of generality we may assume that this function is 1 . One can check that in this case we have $\Phi(1)=1$. It is a folk result that every endomorphism of $C(\Omega)$ which maps 1 into 1 is of the form

$$
f \mapsto f \circ \varphi
$$

with some continuous function $\varphi: \Omega \mapsto \Omega$. Since $\Phi$ is an isometry, by Urysohn's lemma, in our case $\varphi$ is surjective. It remains to prove that $\varphi$ is injective as well. To this end suppose, on the contrary, that there are different points $x, y \in \Omega$ such that $\varphi(x)=\varphi(y)=z$. We construct a continuous function $f: \Omega \rightarrow \mathbb{C}$ in the following way. Let $\left(U_{n}\right)$ be a monotone decreasing sequence of open sets in $\Omega$ such that $\cap_{n} U_{n}=\{z\}$. By Urysohn's lemma, for every $n$ we have a continuous function $f_{n}: \Omega \rightarrow[0,1]$ such that

$$
f_{n}(z)=1 \text { and } f_{n}(t)=0\left(t \in \Omega \backslash U_{n}\right) .
$$

Let

$$
f=1-\sum_{n=1}^{\infty} \frac{1}{2^{n}} f_{n} .
$$


It is easy to see that $f$ is a continuous function and $f(t)=0$ if and only if $t=z$. Since $\Phi$ is a locally surjective isometry, there exists a homeomorphism $\psi: \Omega \rightarrow \Omega$ and a continuous function $\tau: \Omega \rightarrow \mathbb{C}$ having modulus 1 such that

$$
f \circ \varphi=\Phi(f)=\tau \cdot f \circ \psi
$$

It follows that

$$
\{x, y\} \subset(f \circ \varphi)^{-1}(0)=(f \circ \psi)^{-1}(0)=\psi^{-1}(z) .
$$

Since $\psi$ is bijective, this is a contradiction. Consequently, $\varphi$ is injective which implies the surjectivity of $\boldsymbol{\Phi}$.

Remark. As for the general case, we suspect that there exists a "big" compact Hausdorff spaces $\Omega$ with the property the group of all surjective isometries of $C(\Omega)$ is not reflexive. This is only a conjecture, the proof is missing.

\section{Isometries of spin factors}

In this section we prove that for every infinite dimensional Hilbert space the isometry group is algebraically nonreflexive not only with respect to the original Hilbert space norm, but also with respect to the so-called spin norms. Moreover, we give a complete algebraic characterization of the locally surjective isometries of spin factors.

Spin factors are very interesting structures coming from mathematical physics (anticommutation relations) and holomorphy (bounded symmetric domains). In particular, it is a $J B^{*}$-triple (see [6] and [9]). $J B^{*}$-triples form an operator theoretic category which is naturally equivalent to the geometric category of bounded symmetric domains. Every $C^{*}$-algebra is a $J B^{*}$-triple. $B(H)$ and spin factors are examples of type I von Neumann triple factors. The aim of this section is to point out the possible interest of the algebraic reflexivity concept in the theory of $J B^{*}$-triples. It is known that the group of surjective isometries of $B(H)$ is both topologically and algebraically reflexive [13]. We prove below that the corresponding group of a spin factor is not algebraically reflexive and so the algebraic reflexivity distinguishes (to some extent) the type I $J B W^{*}$-factors.

Spin factors can be defined in more than one way. For the purpose of this paper, being operator theoretic in its spirit, we use the following approach.

Let $\mathcal{W}$ be a complex Hilbert space with inner product $(.,$.$) and let x \mapsto \bar{x}$ be a conjugation on it. This means that $-: \mathcal{W} \rightarrow \mathcal{W}$ is a conjugate-linear isometric mapping satisfying $\overline{\bar{x}}=x$. Using polarization, it is easy to see that $\langle\bar{x}, \bar{y}\rangle=\langle y, x\rangle$ holds for all $x, y \in \mathcal{W}$. Every Hilbert space has many conjugations. For example, if $\left\{e_{x}\right\}$ is a complete orthonormal system in $\mathcal{W}$, then for $x=\sum_{x}\left\langle x, e_{x}\right\rangle e_{x}$ we can define $\bar{x}=\sum_{x}\left\langle e_{x}, x\right\rangle e_{x}$. This gives a conjugation on $\mathcal{W}$. 
In what follows, let $\mathcal{W}$ be equipped with a fixed conjugation. One can prove that every system of pairwise orthogonal symmetric unit vectors can be extended to a complete orthonormal family of symmetric elements. Using some elementary computations, we can verify that a norm can be defined on $\mathcal{W}$ by

$$
2\|x\|^{2}=\langle x, x\rangle+\sqrt{\langle x, x\rangle^{2}-|\langle x, \bar{x}\rangle|^{2}} \quad(x \in \mathcal{W}) .
$$

It is easy to see that the norm $\|x\|$ is equivalent to $\sqrt{\langle x, x\rangle}$ and so $(\mathcal{W},\|\|)$ is a Banach space. When we are speaking about metrical properties of spin factors without specific mentioning of the involved norm, we always mean the spin norm $\|$.$\| .$

We define an algebraic operation, called triple product, on $\mathcal{W}$ by

$$
2[x y z]=\langle x, y\rangle z+\langle z, y\rangle z-\langle z, \bar{x}\rangle \bar{y} \quad(x, y, z \in \mathcal{W}) .
$$

Then the $C^{*}$-axiom $\|[x x x]\|=\|x\|^{3}$ is satisfied.

Note also that, when $\operatorname{dim} \mathcal{W} \geq 3, \mathcal{W}$ is algebraically simple with respect to the triple product. A subspace $\mathcal{J} \subset \mathcal{W}$ is called a triple ideal if the condition

$$
[\mathcal{W W J}],[\mathcal{W J W}],[\mathcal{J W W}] \subset \mathcal{J}
$$

holds true. The spin factor $\mathcal{W}$ is said to be algebraically simple if $\{0\}$ and $\mathcal{W}$ are the only ideals of $\mathcal{W}$. Now, let $\operatorname{dim} \mathcal{W} \geq 3$. If $\mathcal{J} \subset \mathcal{W}$ is an ideal and $x \in \mathcal{J}$ is a nonzero element, then for $y \in\{\bar{x}\}^{\perp}$ we have

$$
\langle x, x\rangle y+\langle y, x\rangle x=\langle x, x\rangle y+\langle y, x\rangle x-\langle y, \bar{x}\rangle \bar{x}=2[x x y] \in \mathcal{J}
$$

Since $\langle x, x\rangle \neq 0$, this implies $y \in \mathcal{J}$. Thus $\mathcal{J}$ is of codimension at most one. Since $\operatorname{dim} \mathcal{W} \geq 3$, it follows that $\mathcal{J}$ contains two linearly independent vectors, say $a, b$. By what is proved above, we obtain $\mathcal{J} \supset\{\bar{a}\}^{\perp}+\{\bar{b}\}^{\perp}=\mathcal{W}$ since $\bar{a}, \bar{b}$ are linearly independent. This gives us that $\mathcal{J}=\mathcal{W}$, i.e. $\mathcal{W}$ is algebraically simple.

A linear mapping $\phi: \mathcal{W} \rightarrow \mathcal{W}$ is called a triple homomorphism if

$$
\phi([x y z])=[\phi(x) \phi(y) \phi(z)] \quad(x, y, z \in \mathcal{W}) .
$$

The kernel of a triple homomorphism is always a triple ideal of $\mathcal{W}$. If $\operatorname{dim} \mathcal{W} \geq 3$, it follows that every nonzero homomorphism of $\mathcal{W}$ is injective.

In the sequel we suppose that $\mathcal{W}$ is an infinite dimensional spin factor. We shall characterize the locally surjective isometries of $\mathcal{W}$ as the nonzero triple homomorphisms of $\mathcal{W}$. To this end we need the following lemmas.

Lemma 3.1. Let $u \in \mathcal{W}$ be a nonzero tripotent, i.e. $[u u u]=u$. Then we have either $\langle u, u\rangle=1$ and $\langle u, \bar{u}\rangle=0$ or else $\langle u, u\rangle=2$ and there exists a complex number $\alpha$ of modulus 1 such that $\bar{u}=\alpha u$. 
Proof. For all $u \in \mathcal{W}$ we have $[u u u]=\langle u, u\rangle u-\frac{1}{2}\langle u, \bar{u}\rangle \bar{u}$. If $[u u u]=u$ and $u, \bar{u}$ are linearly independent, then the first possibility holds true. If there exists an $\alpha \in \mathbb{C}$ such that $\bar{u}=\alpha u$, then it is obvious that $|\alpha|=1$ and so $\langle u, u\rangle=2$ follows.

Lemma 3.2. Let $\phi: \mathcal{W} \rightarrow \mathcal{W}$ be a nonzero triple homomorphism. Let $u \in \mathcal{W}$ be such that $\langle u, u\rangle=1$ and $\langle u, \bar{u}\rangle=0$. Then $\langle\phi(u), \phi(u)\rangle=1$ and $\langle\phi(u), \overline{\phi(u)}\rangle=0$.

Proof. Clearly, $u$ is a tripotent. Therefore, $\phi(u)$ is also a tripotent and, by Lemma 3.1, there are two possibilities. Suppose that $\overline{\phi(u)}=\alpha \phi(u),|\alpha|=1$ and $\langle\phi(u), \phi(u)\rangle=2$. Since $\phi([u u \bar{u}])=[\phi(u) \phi(u) \phi(\bar{u})]$, we have

$$
\begin{gathered}
0=\phi(\langle u, u\rangle \bar{u}+\langle\bar{u}, u\rangle u-\langle u, u\rangle \bar{u})= \\
\langle\phi(u), \phi(u)\rangle \phi(\bar{u})+\langle\phi(\bar{u}), \phi(u)\rangle \phi(u)-\langle\phi(\bar{u}), \overline{\phi(u)}\rangle \overline{\phi(u)}= \\
2 \phi(\bar{u})+\langle\phi(\bar{u}), \phi(u)\rangle \phi(u)-\langle\phi(\bar{u}), \alpha \phi(u)\rangle \alpha \phi(u)=2 \phi(\bar{u})
\end{gathered}
$$

and thus $\phi(\bar{u})=0$. But this is impossible since $\phi$ is injective. By Lemma 3.1 we obtain the statement.

Lemma 3.3. Let $\phi, u$ be as in the previous lemma. Then we have $\langle\phi(u), \phi(\bar{u})\rangle=0$.

Proof. We already know from Lemma 3.2 that $\langle\phi(u), \phi(u)\rangle=1$ and $\langle\phi(u), \overline{\phi(u)}\rangle=0$. Since $\phi([u \bar{u} u])=[\phi(u) \phi(\bar{u}) \phi(u)]$ and $2[u \bar{u} u]=2\langle u, \bar{u}\rangle u-\langle u, \bar{u}\rangle u=0$, we have

$$
0=2[\phi(u) \phi(\bar{u}) \phi(u)]=2\langle\phi(u), \phi(\bar{u})\rangle \phi(u)-\langle\phi(u), \overline{\phi(u)}\rangle \overline{\phi(\bar{u})}=2\langle\phi(u), \phi(\bar{u})\rangle \phi(u) .
$$

Therefore, $\langle\phi(u), \phi(\bar{u})\rangle=0$.

Lemma 3.4. Let $\phi: \mathcal{W} \rightarrow \mathcal{W}$ be a nonzero triple homomorphism. Then $\phi$ is an isometry in the Hilbert space norm of $\mathcal{W}$ and thus, it preserves the inner product. Moreover, $\phi$ is of the form $\phi=\beta \psi$, where $|\beta|=1$ and $\psi$ is an isometry with respect to $\langle.,$.$\rangle which preserves the conjugation. In fact, \psi$ is a triple homomorphism as well.

Proof. Let $0 \neq x \in \mathcal{W}$. There exists a 2-dimensional subspace $\mathcal{V} \subset \mathcal{W}$ such that $\overline{\mathcal{V}}=\mathcal{V}$ and $x \in \mathcal{V}$. Indeed, if $x$ and $\bar{x}$ are linearly independent, we may take $\mathcal{V}=\operatorname{span}\{x, \bar{x}\}$. Otherwise we can choose a nonzero vector $x_{1} \in \mathcal{W}$ which is orthogonal to $x$ and satisfies $\overline{x_{1}}=x_{1}$. Then we may define $\mathcal{V}=\operatorname{span}\left\{x, x_{1}\right\}$.

Take $a=\bar{a} \in \mathcal{V}$ with $\langle a, a\rangle=1$. There exists $b \in \mathcal{V}$ which is orthogonal to $a$ and satisfies $\langle b, b\rangle=1$. Since $\bar{b} \in \mathcal{V}$, we have $\bar{b}=\alpha a+\beta b$. From $\alpha=\langle\bar{b}, a\rangle=\langle\bar{b}, \bar{a}\rangle=\langle a, b\rangle=0$ it follows that we may assume $b=\bar{b}$ as well. Now define $u=(a+i b) \sqrt{2}$. It is easy to verify that $u, \bar{u}$ are orthogonal tripotents.

Since $u, \bar{u} \in \mathcal{V}$ and $\mathcal{V}$ is 2-dimensional, there exist $\alpha, \beta \in \mathbb{C}$ such that $x=\alpha u+\beta \bar{u}$. Then 


$$
\langle\phi(x), \phi(x)\rangle=\langle\alpha \phi(u)+\beta \phi(\bar{u}), \alpha \phi(u)+\beta \phi(\bar{u})\rangle
$$

and, by Lemma 3.2 and Lemma 3.3 , we have

$$
\langle\phi(x), \phi(x)\rangle=|\alpha|^{2}\langle\phi(u), \phi(u)\rangle+|\beta|^{2}\langle\phi(\bar{u}), \phi(\bar{u})\rangle=|\alpha|^{2}+|\beta|^{2}=\langle x, x\rangle .
$$

This implies that $\phi$ is an isometry with respect to the Hilbert space norm of $\mathcal{W}$.

Finally, $\phi([x y z])=[\phi(x) \phi(y) \phi(z)]$ gives us that

$$
\begin{gathered}
\langle\phi(x), \phi(y)\rangle \phi(z)+\langle\phi(z), \phi(y)\rangle \phi(x)-\langle\phi(z), \phi(\bar{x})\rangle \phi(\bar{y})= \\
\langle x, y\rangle \phi(z)+\langle z, y\rangle \phi(x)-\langle z, \bar{x}\rangle \phi(\bar{y})=\phi(\langle x, y\rangle z+\langle z, y\rangle x-\langle z, \bar{x}\rangle \bar{y})= \\
\langle\phi(x), \phi(y)\rangle \phi(z)+\langle\phi(z), \phi(y)\rangle \phi(x)-\langle\phi(z), \overline{\phi(x)}\rangle \overline{\phi(y)} .
\end{gathered}
$$

Consequently, we have

$$
\langle\phi(z), \phi(\bar{x})\rangle \phi(\bar{y})=\langle\phi(z), \overline{\phi(x)}\rangle \overline{\phi(y)} .
$$

Hence, if we take some fixed $x \in \mathcal{W}$ with $\langle x, x\rangle=1$, we get

$$
\phi(\bar{y})=\langle\phi(\bar{x}), \phi(\bar{x})\rangle \phi(\bar{y})=\langle\phi(\bar{x}), \overline{\phi(x)}\rangle \overline{\phi(y)}=\alpha \overline{\phi(y)} .
$$

Since $\phi$ is an isometry, the scalar $\alpha$ is of modulus 1 . If $\beta^{2}=\alpha$, then elementary calculation shows that $\psi=\frac{1}{\beta} \phi$ defines a conjugation-preserving triple homomorphism.

Observe that only elementary computations are needed to verify the converse of the above statement. Therefore, we have the following corollary.

Corollary 3.5. A linear mapping $\phi: \mathcal{W} \rightarrow \mathcal{W}$ is a nonzero triple homomorphism if and only if it is of the form $\phi=\beta \psi$, where $|\beta|=1$ and $\psi$ is an isometry with respect to (., .) which preserves the conjugation.

Theorem 3.6. A linear mapping $\phi: \mathcal{W} \rightarrow \mathcal{W}$ is a locally surjective isometry of the infinite dimensional spin factor $\mathcal{W}$ if and only if it is a nonzero triple homomorphism.

Proof. First, let $\phi: \mathcal{W} \rightarrow \mathcal{W}$ be a locally surjective isometry. It is known that every surjective isometry of a spin factor (even that of a $J B^{*}$-triple) is a surjective triple homomorphism [6, 9]. It follows that $\phi$ preserves the tripotents. Let $u \in \mathcal{W}$ be a tripotent satisfying $\langle u, u\rangle=1$ and $\langle u, \bar{u}\rangle=0$ (see Lemma 3.1 ). Then elementary computation shows that $\bar{u}$ is also a tripotent. If $\alpha$ is a complex number of modulus 1 , we set $v=u+\alpha \bar{u}$. Then $\langle v, v\rangle=\langle u, u\rangle+|\alpha|^{2}\langle\bar{u}, \bar{u}\rangle=1+1=2$ and $\bar{v}=\bar{u}+\bar{\alpha} \overline{\bar{u}}=\bar{\alpha}(u+\alpha \bar{u})=\bar{\alpha} v$. It follows that $v$ is a tripotent. Since in this case $\phi(v)$ is also a tripotent, we have $[w w w]=w$ for $w=\phi(u)+\alpha \phi(\bar{u})$. As $w_{1}=\phi(u)$ and $w_{2}=\phi(\bar{u})$ are tripotents, after a rather elementary but tedious expansion of both sides of $[w w w]=w$, we obtain that 


$$
0=2 \alpha\left[w_{1} w_{1} w_{2}\right]+\bar{\alpha}\left[w_{1} w_{2} w_{1}\right]+2\left[w_{2} w_{2} w_{1}\right]+\alpha^{2}\left[w_{2} w_{1} w_{2}\right]
$$

Inserting $\alpha= \pm 1, \pm i$, we get

$$
\left[w_{1} w_{1} w_{2}\right]=\left[w_{1} w_{2} w_{1}\right]=\left[w_{2} w_{2} w_{1}\right]=\left[w_{2} w_{1} w_{2}\right]=0
$$

Now take an arbitrary $x \in \mathcal{W}$. From the first three paragraphs of the proof of Lemma 3.4 we can learn that $x$ is of the form $x=\alpha u+\beta \bar{u}$ where $u, \bar{u}$ are tripotents satisfying $\langle u, u\rangle=\langle\bar{u}, \bar{u}\rangle=1,\langle u, \bar{u}\rangle=0$ and $\alpha, \beta$ are scalars. Denote $w_{1}=\phi(u)$ and $w_{2}=\phi(\bar{u})$. Using (11) we have

$$
\begin{gathered}
{[\phi(x) \phi(x) \phi(x)]=\left[\left(\alpha w_{1}+\beta w_{2}\right)\left(\alpha w_{1}+\beta w_{2}\right)\left(\alpha w_{1}+\beta w_{2}\right)\right]=} \\
\alpha|\alpha|^{2}\left[w_{1} w_{1} w_{1}\right]+\beta|\beta|^{2}\left[w_{2} w_{2} w_{2}\right]=\alpha|\alpha|^{2} w_{1}+\beta|\beta|^{2} w_{2}=\alpha|\alpha|^{2} \phi(u)+\beta|\beta|^{2} \phi(\bar{u}) .
\end{gathered}
$$

Now we compute $[x x x]$ and $\phi([x x x])$. We have

$$
2[u u \bar{u}]=\langle u, u\rangle \bar{u}+\langle\bar{u}, u\rangle u-\langle u, \overline{\bar{u}}\rangle \bar{u}=\bar{u}-\bar{u}=0
$$

and in a similar way $[u \bar{u} u]=[\bar{u} \bar{u} u]=[\bar{u} u \bar{u}]=0$. Thus $[x x x]=\alpha|\alpha|^{2} u+\beta|\beta|^{2} \bar{u}$ and so (12) implies $\phi([x x x])=[\phi(x) \phi(x) \phi(x)]$. Similarly to the last part of the proof of Lemma 1.2, after linearization it follows that $\phi$ is a triple homomorphism. This finishes the proof of the first implication.

In order to prove the converse direction, we must verify that every nonzero triple homomorphism of $\mathcal{W}$ is a locally surjective isometry of $\mathcal{W}$. So, let $\phi$ be a nonzero triple homomorphism. Using Lemma 3.4, we may assume that $\phi$ preserves the conjugation, i.e. $\overline{\phi(x)}=\phi(\bar{x})$ holds for every $x \in \mathcal{W}$.

Take $x \in \mathcal{W}$. Since $x=\frac{1}{2}(x+\bar{x})+i \frac{1}{2 i}(x-\bar{x})$, there exist symmetric unit vectors $h, k \in \mathcal{W}$ and real numbers $\gamma, \delta$ such that $x=\gamma h+i \delta k$. Suppose that $h, k$ are linearly independent. We have $\langle h, k\rangle=\langle\bar{h}, \bar{k}\rangle=\langle k, h\rangle$ and then it follows that $\langle h, k\rangle \in \mathbb{R}$. Consequently, the vector $l_{1}=k-\langle k, h\rangle h \neq 0$ satisfies $\bar{l}_{1}=l_{1}$. Thus $k$ can be written as an orthogonal sum $k=\alpha h+\beta l$ with some symmetric unit vector $l$ which is orthogonal to $h$. This shows that we can form complete orthonormal systems $\left\{h, l, e_{x}, \ldots\right\}$ and $\left\{\phi(h), \phi(l), f_{\alpha}, \ldots\right\}$ consisting of symmetric vectors. Recall that since $\phi$ is a nonzero triple homomorphism, it preserves the inner product of $\mathcal{W}$. We define a surjective inner product preserving mapping $U: \mathcal{W} \rightarrow \mathcal{W}$ by $U(h)=\phi(h), U(l)=\phi(l)$ and $U\left(e_{x}\right)=f_{x}$ for every $\alpha$. It is easy to see that $U(\bar{x})=\overline{U(x)}$ for all $x \in \mathcal{W}$. One can verify by a short computation that every conjugation preserving isometry with respect to the Hilbert space norm of $\mathcal{W}$ is isometric with respect to the spin norm as well. Since $x$ is a linear combination of $h, l$ and $U$ equals $\phi$ on $\{h, l\}$, we obtain that $\phi(x)=U(x)$ where $U$ is a surjective isometry of $\mathcal{W}$.

The case when $h$ and $k$ are linearly dependent can be treated in a similar but even simpler way.

Finally, the above statements result in the following theorem. 
Theorem 3.7. Let $\mathcal{W}$ be an infinite dimensional spin factor. Then the group of all surjective isometries of $\mathcal{W}$ is algebraically nonreflexive.

Proof. If $\phi: \mathcal{W} \rightarrow \mathcal{W}$ is a nonsurjective isometry with respect to the Hilbert space norm which preserves the conjugation (see the construction of $U$ above), then $\phi$ is a triple homomorphism and hence a nonsurjective locally surjective isometry of $\mathcal{W}$.

We wish to close this section with some remarks on general $J B^{*}$-triples. If such a triple $\mathcal{W}$ contains a direct summand which is isomorphic to an infinite dimensional spin factor, then its isometry group is not algebraically reflexive. This is easy to see since in $\mathcal{W}=\mathcal{W}_{0} \oplus \operatorname{Spin}(H)$ the sum of $\mathrm{id}_{\mathcal{W}_{0}}$ and an injective triple homomorphism of $\operatorname{Spin}(H)$ is again an injective triple homomorphism and consequently an isometry.

In general $J B^{*}$-triples one should turn attention, we believe, to the representation theory. Every extreme point of a dual ball of $\mathcal{W}$ generates a type I representation. These representations come in 6 generic types; rectangular, simplectic, hermitian, spin and two exceptional. The rectangular factor has a reflexive isometry group. The methods of this paper and some known results about isometries of simplectic and hermitian factor, as well as associated $C_{p}$ classes, should be sufficient to prove that their isometry groups are reflexive. The exceptional factors are finite dimensional and so their isometry groups are also reflexive.

It is therefore reasonable to expect that nonreflexivity of the isometry group of $\mathcal{W}$ indicates the presence of infinite dimensional spin representations. There is at this moment a, seemingly nontrivial, obstacle in our attempt to verify such a conjecture. If $\mathcal{W}$ has an infinite dimensional spin representation, then its second dual, which is also a $J B^{*}$-triple, contains spin factor as its direct summand and hence its isometry group is nonreflexive. However, this does not trivially imply that the isometry group of $\mathcal{W}$ is also nonreflexive. The verification of this conjecture should be the cornerstone of general theory of reflexivity of isometry groups of $J B^{*}$-triples, which we plan in the near future.

\section{Open problems}

The algebraic properties of surjective isometries of Banach algebras attracted the attention of many mathematicians (see [6] and the references therein). We therefore think that it would be worthwhile to study the problem of reflexivity of the group of surjective isometries in the setting of general Banach spaces and examine its relations to the geometry of Banach spaces.

We collect below some problems which we think are of interest.

Problem 1. Let $X$ be an infinite dimensional Banach space which is linearly homeomorphic to a Hilbert space. Is the group of all of its surjective isometries algebraically nonreflexive?

We think that the answer to this problem is affirmative. 
Problem 2. What is the relation between the algebraic reflexivity of the isometry groups of $X$ and $X^{*}$ ?

Problem 3. Let $\mathcal{A}$ be an infinite dimensional von Neumann factor acting on a separable infinite dimensional Hilbert space. Is the group of its surjective isometries algebraically reflexive?

Acknowledgement. This paper has been written during the first author's stay at the University of Maribor, Slovenija. He is very grateful to his hosts for their kind hospitality and to Soros Foundation for supporting this visit.

\section{REFERENCES}

1. J. Arazy, The isometries of $C_{p}$, Israel J. Math. 22 (1975), 247-256.

2. W. B. Arveson, Continuous analogues of Fock spaces, Mem. Amer. Math. Soc. 80 (1989), 409.

3. M. BREŠAR, Characterization of derivations on some normed algebras with involution, $J$. Algebra 152 (1992), 454-462.

4. M. BREŠAR and P. ŠEMRL, Mappings which preserve idempotents, local automorphisms, and local derivations, Canad. J. Math. 45 (1993), 483-496.

5. M. Brešar and P. ŠEMrL, On local automorphisms and mappings that preserve idempotents, Studia Math. 113 (1995), 101-108.

6. T. Dang, Y. Friedman and B. Russo, Affine geometric proofs of the Banach Stone theorems of Kadison and Kaup, Rocky Mountain J. Math. 20 (1990), 409-428.

7. J. A. ERdos, A simple proof of Arazy's theorem, Proc. Edinburgh Math. Soc. 37 (1994), 239-242.

8. R. V. Kadison, Local derivations, J. Algebra 130 (1990), 494-509.

9. W. KAUP, A Riemann mapping theorem for bounded symmetric domains in complex Banach spaces, Math. Z. 183 (1983), 503-529.

10. D. R. Larson, Reflexivity, algebraic reflexivity and linear interpolation, Amer. J. Math. 110 (1988), 283-299.

11. D. R. LARSON and A. R. Sourour, Local derivations and local automorphisms of $B(X)$ (Proc. Sympos. Pure Math. 51, Part 2, Providence, Rhode Island 1990), 187-194.

12. A. J. Loginov and V. S. Shul'man, Hereditary and intermediate reflexivity of $W^{*}$ algebras, Izv. Akad. Nauk SSSR 39 (1975), 1260-1273; English transl. in USSR-Isv. 9 (1975), 1189-1201.

13. L. MolnáR, The set of automorphisms of $B(H)$ is topologically reflexive in $B(B(H))$, preprint.

14. L. MOLNÁR and B. ZaLAR, On automatic surjectivity of Jordan homomorphisms, Acta Sci. Math. (Szeged) 61 (1995), 413-424.

15. H. L. Royden, Real Analysis (Macmillan, New York, 1963).

16. B. Russo and H. A. DYe, A note on unitary operators in $C^{*}$-algebras, Duke Math. J. 33 (1966), 413-416. 
17. V. S. Shul'man, Operators preserving ideals in $C^{*}$-algebras, Studia Math. 109 (1994), $67-72$.

18. A. R. Sourour, Isometries of norm ideals of compact operators, J. Funct. Anal. 43 (1981), 69-77.

19. E. Størmer, On the Jordan structure of $C^{*}$-algebras, Trans. Amer. Math. Soc. 120 (1965), 438-447.

INSTITUTE OF MATHEMATICS

LAJOS KOSSUTH UNIVERSITY

4010 Debrecen, P.O. BoX 12

HuNGaRY

E-mail address: molnarl@math.klte.hu
Department of Basic Sciences

FaCULTY OF CIVIL ENGINEERING

UNIVERSITY OF MARIBOR

SMETANOVA 17

62000 MARIBOR

SLOVENIJA

E-mail address: borut.zalar@uni-mb.si or borut.zalar@uni-lj.si 